\title{
A pan-transcriptome analysis shows that disease resistance genes have undergone more selection pressure during barley domestication
}

\author{
Yanling Ma', Miao Liu², ${ }^{1,2}$ Jiri Stiller ${ }^{1}$ and Chunji Liư ${ }^{1 *}$
}

\begin{abstract}
Background: It has become clear in recent years that many genes in a given species may not be found in a single genotype thus using sequences from a single genotype as reference may not be adequate for various applications.

Results: In this study we constructed a pan-transcriptome for barley by de novo assembling 288 sets of RNA-seq data from 32 cultivated barley genotypes and 31 wild barley genotypes. The pan-transcriptome consists of 756,632 transcripts with an average N50 length of $1240 \mathrm{bp}$. Of these, 289,697 (38.2\%) were not found in the genome of the international reference genotype Morex. The novel transcripts are enriched with genes associated with responses to different stresses and stimuli. At the pan-transcriptome level, genotypes of wild barley have a higher proportion of disease resistance genes than cultivated ones.

Conclusions: We demonstrate that the use of the pan-transcriptome dramatically improved the efficiency in detecting variation in barley. Analysing the pan-transcriptome also found that, compared with those in other categories, disease resistance genes have gone through stronger selective pressures during domestication.
\end{abstract}

Keywords: Barley, Pan-transcriptome, Novel transcripts, Wild, Cultivated, Resistance

\section{Background}

The phenomenon that an individual contains only a proportion of the genes in a given species was initially noticed in microbes [1]. This phenomenon led to the concept of pan-genome which consists of core and dispensable genomes $[2,3]$. The core component contains genes shared by all individuals of a given species and the remainder belong to the dispensable component [3]. Reports on pan-genome for major crop species appeared only in recent years. Similar to that observed in various microbes, large proportions of genes in different crop species have also been found to be dispensable. For example, dispensable genes account for about $20 \%$ of the genomes in soybean (Glycine soja) [4] and Brassica oleracea [5], 36\% in bread wheat (Triticum aestivum) [6,7],

\footnotetext{
* Correspondence: chunji.liu@csiro.au

${ }^{1}$ CSIRO Agriculture \& Food, 306 Carmody Road, St Lucia, QLD 4067, Australia Full list of author information is available at the end of the article
}

$50 \%$ in maize (Zea mays) $[8,9]$ and $43 \%$ in rice (Oryza sativa L.) [10].

Cultivated barley (Hordeum vulgare ssp. vulgare), derived from its wild progenitor $H$. vulgare spp. spontaneum, is an ancient crop that was domesticated about 10,000 years ago in the Fertile Crescent. It is an important crop growing in highly diverse environments, and has been widely used as human food, animal feed and for fermented and distilled beverages [11]. Barley is a diploid and inbreeding species with a large haploid genome of 5.1 gigabases $(\mathrm{Gb})$ and about $80 \%$ of the genome is characterised by repetitive elements and large pericentromeric regions that are virtually devoid of meiotic recombination [11, 12]. Even with the rapid progress in sequencing technique and capacity, it is still challenging to generate a high quality genome assembly for species like barley. After more than a decade of dedicated efforts, an international team consisting of over 70 researchers have successfully sequenced the complete 
genome of barley based on the cultivar Morex [12]. It seems unlikely that high quality genome assemblies for a large number of barley genotypes can be obtained in the near future. Thus a high quality pan-genome of barley may have to wait. However, capturing the majority of the expressed genes in a barley genotype is now relatively easy and inexpensive. Transcriptome profiling based on RNA-seq has in recent years become the technique of choice in capturing different species of transcripts including mRNAs, non-coding RNAs and small RNAs [13]. RNA-seq data from a wide range of studies with different objectives are now available for not only cultivated but also wild barley genotypes. They include studies varying from stress tolerance [14-18] to domestication [19, 20] and plant development [21, 22]. The availability of such a large quantity of RNA-seq datasets provides a good opportunity to construct a pan-transcriptome to capture most of expressed genes in barley genome.

\section{Methods}

\section{Collection of RNA-seq data}

A total of 288 sets of RNA-seq data from 63 barley genotypes were collected for this study. Of these, 234 were derived from 32 cultivated genotypes (Hordeum vulgare ssp. vulgare) and 54 from 31 wild accessions (Hordeum vulgare ssp. spontaneum) (Additional file 1: Table S1). The data consisted of a total of 6,321,262,514 reads downloaded from the EMBL (European Molecular Biology Lab)/EBI (European Bioinformatics Institute)-European Nucleotide Archive (ENA) database and the National Center for Biotechnology Information (NCBI) Short Sequence Read Archive (SRA) database. The sequences were obtained from a wide range of studies on different environmental factors and stress treatments including low temperature (vernalisation) [23, 24], photoperiod [25], drought [15], salinity [14, 17, 26], heat stress [18], disease infection [NCBI-GEO (Gene Expression Omnibus) accession GSE83676] and excessive nutrients [16]. Some of the sequences were obtained from studies on domestication [19, 20], tissue development ([21, 22], NCBI-GEO accession GSE87377) and whole genome sequencing in barley [11].

\section{Transcriptome reconstruction}

Trimming and filtering of raw RNA reads were performed with SolexaQA++ software [27]. As the average spot lengths (AvgSpotLen) of different RNA-seq datasets were not the same, different filtering standards were applied to exclude low quality reads (Additional file 2: Table S2). Using Trinity (version 2.0.6) [28] with K-mers $=25$, cleaned RNA-seq reads were pooled together and de novo assembled with a minimum transcript length of 200 bp for all three assemblies: cultivated+wild-assembly
(CWA, including all 63 genotypes), cultivated-assembly (CA, including only cultivated genotypes), and wild-assembly (WA, including only wild genotypes). Contaminated transcripts were checked using the stand-alone DeconSeq toolkit (version 0.4.3) [29] with the default parameters. Bacterial genomes, viral genomes and human genomes downloaded from NCBI were used to build the 'remove' databases which were used to identify contaminant transcripts. Plant genomes of maize, rice, wheat, soybean (Glycine max), sorghum (Sorghum bicolor), Arabidopsis thaliana, Brachypodium distachyon and Medicago truncatula were downloaded from EnsemblPlants database and used to build the 'retain' databases.

\section{Identification of novel transcripts not present in Morex}

Transcripts from the three assemblies were aligned to the Morex genome sequences (the international reference genome for barley) using the GMAP programme [30]. They were then mapped against the cDNAs (ASM32608V1.31 from EnsemblPlants database), high-confidence $(\mathrm{HC})+$ low-confidence (LC) transcripts and $\mathrm{HC}+\mathrm{LC}$ genes of Morex [12] using Blastn (version 2.2.28+). Transcripts with similarity $<85 \%$ and coverage $<85 \%$ were retrieved and defined as novel. They were then clustered using the cd-hit-est programme from cd-hit package v4.6.4 [31] based on sequence similarity $(\mathrm{c}=0.95)$.

\section{Functional annotation of transcripts}

ORFs (open reading frames) of transcripts were predicted using TransDecoder v3.0.0 (https://github.com/ TransDecoder/TransDecoder/releases) with a minimum length of $300 \mathrm{nt}$ (100 amino acids). Redundant CDS (coding DNA sequence) were removed using the cd-hit-est program $(c=0.98)$. For CDS from the novel transcripts, non-redundant CDS were aligned back to the reference sequences of Morex and barley Unigene database (ftp://ftp.ncbi.nih.gov/repository/UniGene/Hordeum_vulgare/), and CDS without significant hits (similarity $<85 \%$ and coverage $<85 \%$, E-value $\leq 1 \mathrm{e}-6$ ) were retained. GO (gene ontology) classification for biological process was conducted by searching against plant proteins databases using the AgBase Goanna programme and summarized by GOSlimViewer with the default parameters [32]. GO enrichment analysis was performed with agriGov2.0 [33] and REVIGO [34]. HMMER v3.1b2 software [35] was used to detect Pfam-A domains (Pfam31.0) with E-value $\leq 1 \mathrm{e}-3$ [36]. NLR-parser [37] was applied to predict the NLR (nucleotide-binding leucine-rich repeat)-associated motifs and detect the NBS-LRR (nucleotide-binding site leucine-rich repeat) type disease resistance genes.

Those novel transcripts without any predicted ORFs were retrieved. They were aligned against the barley EST database (expressed sequence tag, B-EST, v2.1), fulllength cDNAs of cv. Haruna Nijo [38] and barley 
UniGene database using Blastn with a minimum similarity of $90 \%$ and coverage $90 \%$ (E-value $\leq 1 \mathrm{e}-6$ ). The remaining unaligned transcripts were queried against proteins from the UniProtkb_Viridiplantae database (EMBL-EBI) and the AgBase plant protein database [32]. Alignments with a minimum identity and coverage both at $70 \%$ were considered as significant matches (E-value $\leq 1 \mathrm{e}-6)$. Those transcripts without any significant hits to the protein databases were aligned against ncRNA sequences from the NONCODE database [39] and long ncRNAs of Morex [12] using Blastn (E-value $\leq 1 \mathrm{e}-6$ ) and the Rfam database [40] with the Infernal software version 1.1.2 [41].

\section{SNP discovery}

Trimmed reads of the representative RNA-seq datasets (dataset with the largest reads number when there are replicates for a specific accession) for each genotype were mapped to the pan-transcriptome (CWA) with Bowtie2 (v2.2.9) [42]. Redundant sequences of the CWA were firstly removed using cd-hit-est program $(c=0.95)$ before reference building. Duplicated reads were removed using the samtools v1.3.1 from the SAMtools package [43]. SNPs (single nucleotide polymotphisms) or SNVs (single nucleotide variants) were called by using 'samtools mileup' and 'bcftools call' [43] commands with MAPQ $\geq 20$ and were filtered with bcftools ( - e 'DP $<4$ ' --SnpGap 3). The variant rates for cultivated and wild barley genotypes were annotated with SnpEff v $4.3 \mathrm{t}$ [44]. Based on the gmap results with the Morex genome, SNP density along each chromosome was calculated with a $10 \mathrm{Mb}$ window size. Based on SNP data from 63 cultivated and wild barley genotypes, the principle component analysis (PCA) was conducted using vcftools v0.1.14 [45] (converting the SNP vcf files to .ped and .map files), plink v1.90 beta [46] (converting .ped and .map files to binary files) and GCTA v1.91.7 beta [47] (outputting the .eigenval and .eigenvec files). The first two principal components were selected for categorizing the cultivated and wild barley genotypes.

\section{Genetic differentiation between cultivated and wild barley}

To estimate the genetic differentiation of cultivated and wild barley, the patterns of allele frequency for each locus were measured using the SNP data from the two groups of genotypes. Gene differentiation was measured by the fixation index $\left(\mathrm{F}_{S T}\right)$ using vcftools v0.1.14 [45]. Transcripts with $\mathrm{F}_{S T}$ larger than the 95th percentile were treated as having been subjected to strong selective pressures. Distribution of such transcripts on each chromosome was investigated by alignment them against the Morex genome. The KEGG (Kyoto Encyclopedia of Genes and Genomes) internal annotation tool BlastKOALA (www.kegg.jp/blastkoala/) and KAAS (https://www.genome.jp/tools/kaas/) were used to assign $\mathrm{K}$ numbers to those transcripts and enriched pathways were identified by KEGG mapping (www.kegg.jp/kegg/mapper.html).

We also analysed the barley Mla (Mildew resistance locus a) gene families which are specific to resistance to powdery mildew and genetic variations of the Mla locus found among cultivars [48]. Since the Mla locus on chromosome $1 \mathrm{H}$ has been subjected to extreme functional diversification and it encodes by far the largest number of R genes [49], it was selected for this study. Thirty-one published gene sequences of this locus were blast against CWA, CA and WA (identity $\geq 90 \%$, coverage $\geq 50 \%$ ) to identify matched transcripts. The $\mathrm{F}_{S T}$ value the Mla gene transcripts from CWA was extracted and the functional domains of the top hits from CA and WA were analysed by searching against NCBI Conserved Domains Databases (CDD) [50] with default settings.

\section{Results \\ Classification and functional analysis of novel transcripts not present in Morex}

The total number of transcripts from the cultivated +wild-assembly (CWA) were 756,632 with a N50 length of $1240 \mathrm{bp}$. When analysed against the Morex genome sequences, cDNAs, $\mathrm{HC}+\mathrm{LC}$ transcripts and $\mathrm{HC}+\mathrm{LC}$ genes, 289,697 of the transcripts $(38.2 \%$, with a total length of $203.9 \mathrm{Mbp}$ ) were identified as novel (Fig. 1a). Following the removal of the redundant sequences (identity threshold 95\%), 235,887 representative (non-redundant) transcripts were retained. Of the novel CDS, 86,383 were identified with predicted open reading frames (ORFs) with a minimum of $300 \mathrm{nt}$ (100 amino acids). Aligning the coded protein sequences to the Pfam database identified 54,090 CDS with Pfam domains (Fig. 1a). Numbers of top assigned domains and domains related to environmental stresses were counted (Additional file 3: Figure S1). Compared with genes in Morex, 15 types of protein domains were substantially enriched (percentage difference $\geq 1.5$ times) in the novel CDS. They include peptidase, LRR domain, ABC transporter, PPR (pentatricopeptide repeat) domain, shortchain dehydrogenase, exchangers/symporters and salt stress/antifungal family proteins. Meanwhile, significant hits with known plant proteins were found for 44,559 of the novel CDS, and GO annotations were assigned to 22,620 of them (Fig. 1a). GO classification for biological processes indicates a large proportion of the novel CDS was involved with 'response to biotic and abiotic stimuli' and 'defense response'. These GO terms were all significantly enriched in comparison with the genes in Morex $(P<0.05)$ (Fig. 2). Aligning those transcripts without predicted ORFs against the barley EST database, the fulllength cDNAs of cv. Haruna Nijo and the barley UniGene database found 545 transcripts with 


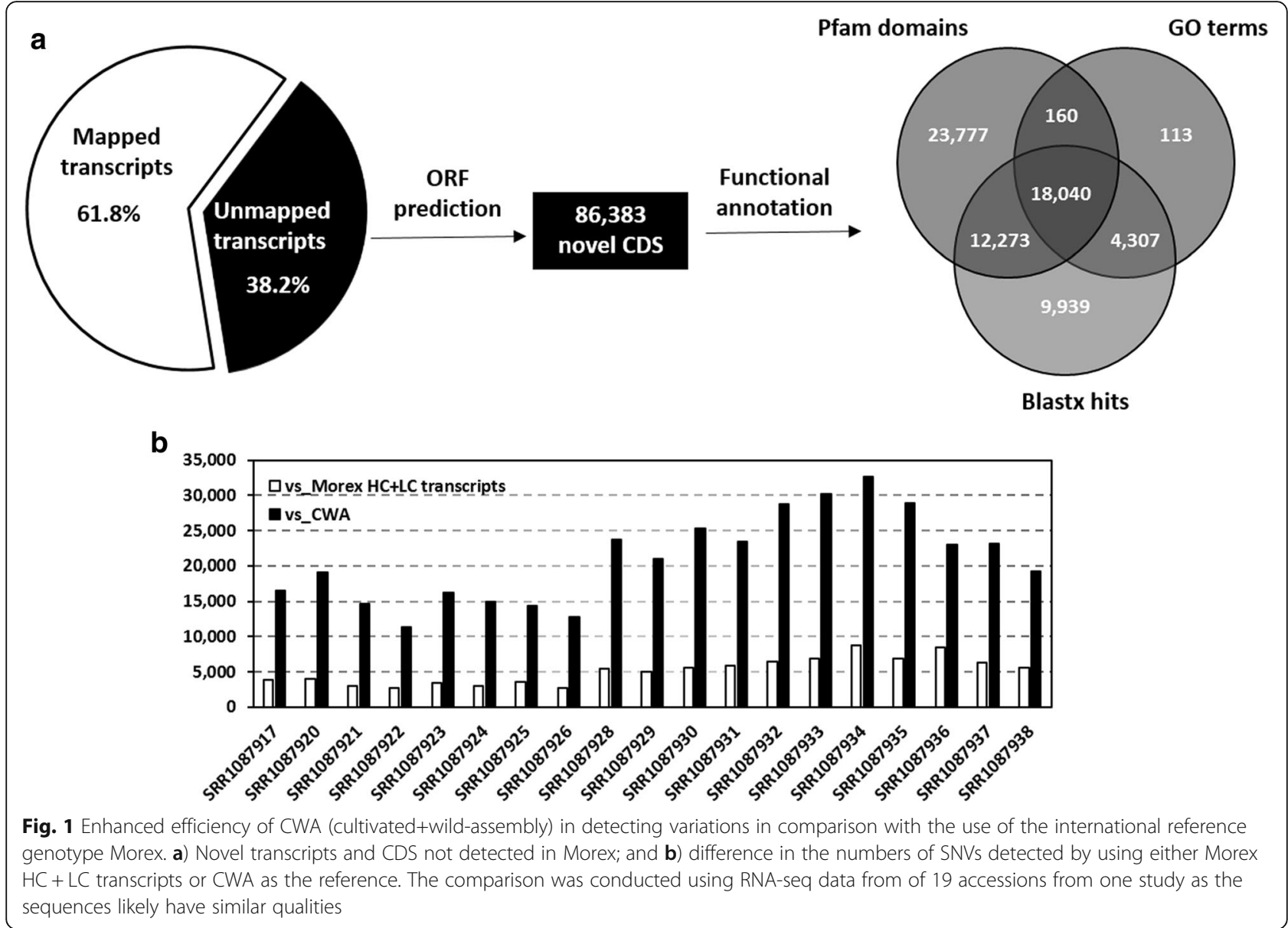

significant hits. A total of 23,556 of the remaining transcripts had significant hits to known protein databases. For those transcripts without significant hits to known proteins, 7018 detected significant matches with ncRNA (non-coding RNA) sequences.

To evaluate its efficiency in detecting genetic variations, the CWA (containing 528,646 transcripts after redundancy removal) were used as the reference for reads mapping. Trimmed RNA-seq reads of 19 cultivated and wild genotypes from a single study [20] were used for this assessment. The reads were mapped back to Morex $\mathrm{HC}+\mathrm{LC}$ transcripts and CWA, respectively. Compared with the use of the Morex $\mathrm{HC}+\mathrm{LC}$ transcripts (SNVs ranging from 2655 to 8753), approximately four times more SNVs were detected when CWA (SNV number ranging from 11,408 to 32,610 ) was used as the reference. The mapping percentage of the reads also increased from 95.9 to $98.5 \%$ when CWA was used as the reference (Fig. 1b; Additional file 4: Table S3).

\section{Functional comparison of transcripts from CA and WA}

A total of 665,270 and 220,990 transcripts were assembled for cultivated (CA) and wild barley (WA), respectively. Of them, a total of 80,354 novel CDS for CA and 3929 for WA were identified (Fig. 2). We also found $\sim 60 \%$ transcripts of CA are absent in WA and 16\% transcripts of WA are not present in CA. The large difference in the number of novel CDS and the proportion of common transcripts identified between cultivated and wild barley is likely due to the less diverse tissue types used (Additional file 1: Table S1) and the much smaller quantity of sequences $(\sim 157 \mathrm{~Gb}$ compared with $\sim 796 \mathrm{~Gb}$ for cultivated barley) used for the wild barley genotypes. Classification of GO terms in biological process suggested a large number of the novel CDS from the wild barley were involved in 'response to stress', 'multicellular organism development,' cellular component organization,',post embryonic development', 'reproduction' and 'anatomical structure morphogenesis', 'growth', 'cell differentiation and cell growth'. These GO terms all had substantially higher percentages (number of proteins for a specific GO term divided by the total protein number with $\mathrm{GO}$ terms $\geq 1.5$ times) than those from the cultivated barley. The percentages of GO terms 'transport,' 'biosynthetic process', 'embryo/ flower development,' 'lipid/carbohydrate/secondary metabolic process' and 'regulation of gene expression' are substantially higher for novel CDS from the cultivated barley. 


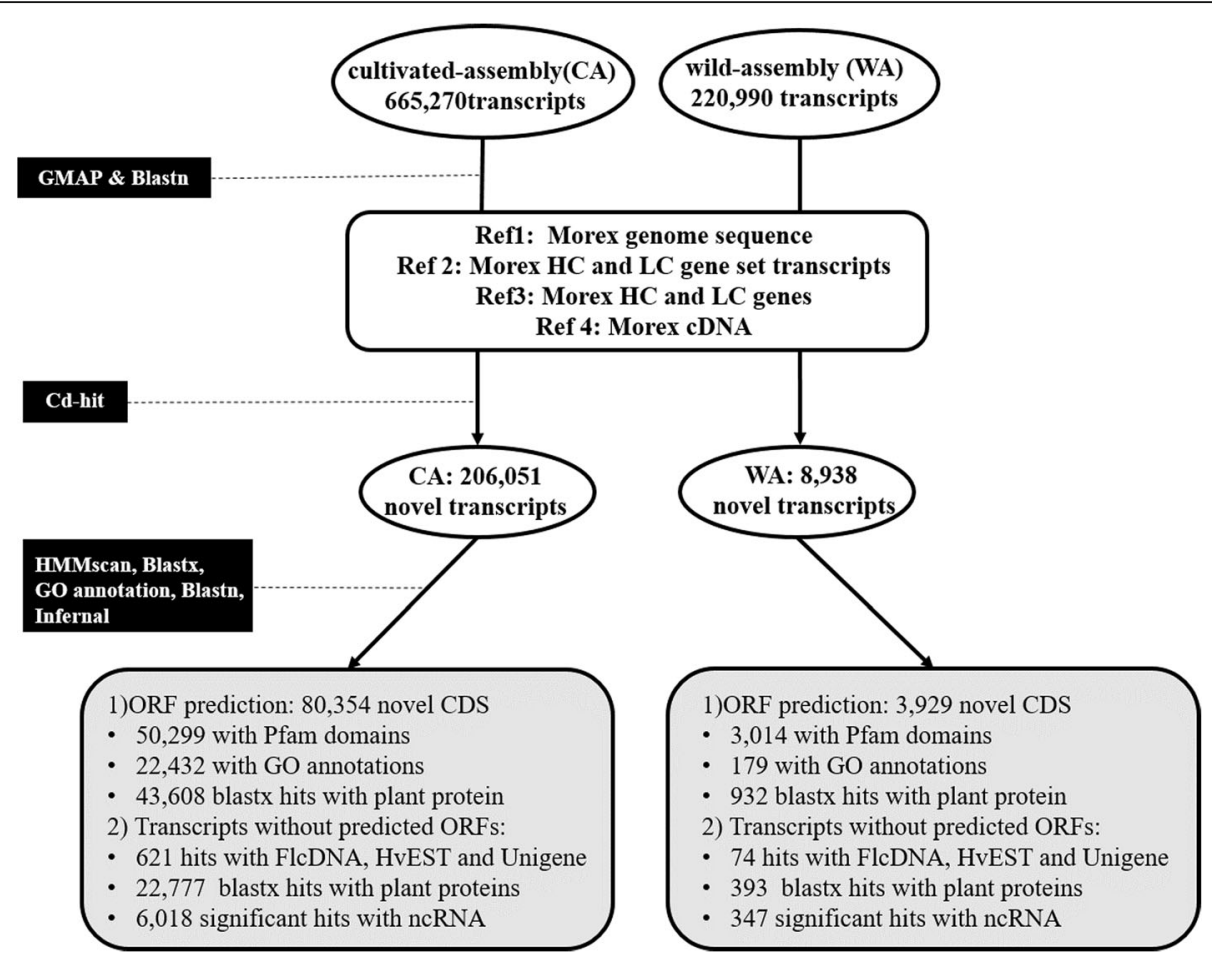

Fig. 2 The workflow for identification and annotation of novel transcripts from cultivated and wild barley genotypes

Pfam domains were assigned to 50,299 of the novel CDS from the cultivated and 3014 of those from the wild barley genotypes (Fig. 3). The proportions of several protein classes, including LRR, NB-ARC (nucleotidebinding adaptor shared by APAF-1, $\mathrm{R}$ proteins, and CED-4), elongation factor, HSP (heat shock protein) /HSF (heat shock factor) protein, EF-hand, wall-associated kinase (WAK) and 'cold-shock' DNA-binding domain-containing proteins, are more than 1.5 times higher in the wild barley than those in cultivated genotypes (Fig. 3a). Similar analysis of CDS from all transcripts of CA and WA showed that the genes with LRR, NB-ARC, WAK, Myb/SANT (SWI3, ADA2, N-CoR, and TFIIIB)-like DNA-binding domain, LEA (late embryogenesis abundant) and plant mobile domains are more abundant in wild barley genotypes than those of cultivated ones (Fig. 3b). Apart from the Pfam annotation, the NLR-parser analysis also showed that both novel CDS and the whole CDS set of wild barley had a significantly higher proportion (two to five fold higher) of NLR genes (including both CNL and TNL genes) than those of cultivated barley (Table 1).

Genetic diversity of wild barley and cultivated barley with SNP discovery

Using CWA as reference, a total of 441,770 SNPs were identified among the cultivated barley genotypes and 672,579 SNPs were detected among the wild barley genotypes. The number $(485,473)$ and proportion (72.2\%) of unique SNPs were significantly higher in the wild barley genotypes than those in the cultivated ones (254,664, 57.6\%). SnpEff annotation analysis also found higher genetic diversity among the wild barley genotypes (1/193, one variant for every 193 bases) than that among the cultivated ones (1/144). The distribution of SNPs along each of the chromosomes is visualized with a 10 $\mathrm{Mb}$ window size (Additional file 5: Figure S2). A typical $\mathrm{V}$-shaped distribution pattern was observed for all chromosomes. Chromosome $7 \mathrm{H}$ shows the largest difference in SNP density (with an average of 1.8 times) between cultivated and wild barley genotypes. PCA analysis indicated the SNP data can effectively discriminate the cultivated and wild barley. They were clustered into two distinct groups with no obvious overlapping although five of the cultivated barley accessions show large genetic distances from the others (Additional file 6: Figure S3).

\section{Disease resistance genes suffered more selective pressures during barley domestication}

With the 95th percentile of $F_{S T}$ values $(0.72)$ as the threshold, a total of 6520 transcripts were found under strong selection pressures (Fig. 4a). These transcripts are distributed unevenly between different chromosomes although the 'V-shaped' distribution is conserved for all chromosomes (Fig. 4b-h). Chromosome $3 \mathrm{H}$ possesses the largest number of genes under strong selection while 


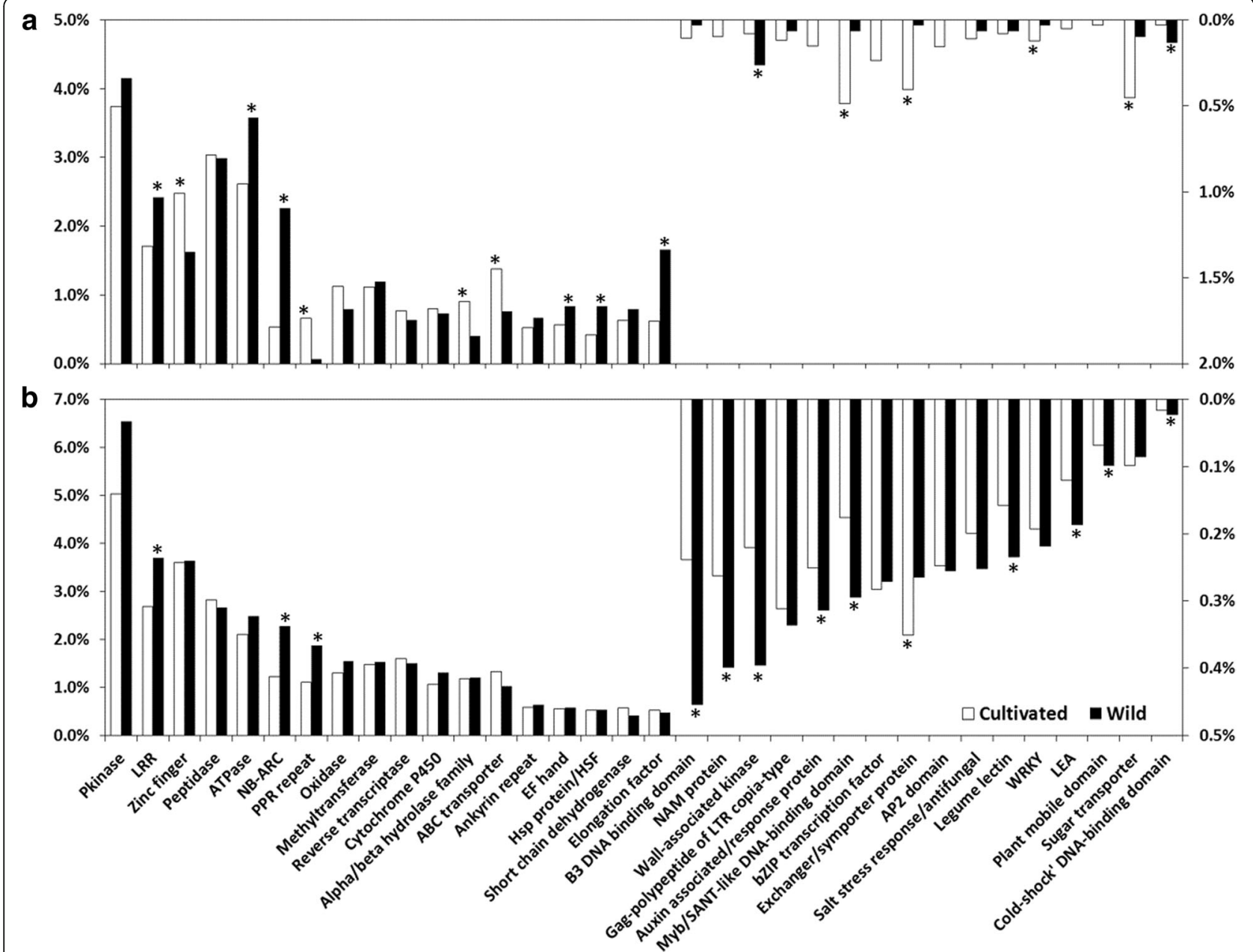

Fig. 3 Difference in annotated protein domains between cultivated and wild barley. a Difference in the numbers of novel CDS. $\mathbf{b}$ Difference between all predicted CDS. The Y-axis shows the percentage of CDS with a specific protein domain. Protein domains marked with * indicate significant difference between cultivated and wild barley

there is little difference between the other six chromosomes. GO enrichment analysis on the 3165 genes predicted from these transcripts found that they are heavily involved in biological processes including 'response to stress', 'response to abiotic/biotic stimulus', 'development process,' 'anatomical structure development', 'regulation of biological process' and 'secondary metabolic process' (Fig. 5a). When analysed against the KEGG pathway database, these genes were found to be enriched in pathways of biosynthesis of antibiotics, plant-pathogen interaction, phenylpropanoid biosynthesis and plant hormone signal transduction (Fig. 5b).

The blast analysis of the 31 variants of Mla locus against CWA identified eight matched transcripts and only two of them had $\mathrm{F}_{S T}$ values: 0.49 for TR1 20931_c5_g1_i1and 0.53 for TR120931_c5_g1_i6. This indicate the Mla gene families have still been under high selection pressure. When separating cultivated and wild barley genotypes, the top CA hits of Mla genes are TR100774_c1_g1_(i2, i3, i5, i6,i7), all of which have

Table 1 Difference in NLR genes identified between cultivated and wild barley with NLR-parser analysis

\begin{tabular}{llllll}
\hline CDS sets & CC $^{\mathrm{a}}$-NBS-LRR(CNL) & TIR $^{\mathrm{b}}$-NBS-LRR(TNL) & Number of NLR genes & Number of total CDS/genes & Percentage of NLR genes \\
\hline novel CDS of CA & 114 & 58 & 172 & 80,354 & $0.21 \%$ \\
novel CDS of WA & 39 & 1 & 40 & 3929 & $1.02 \%$ \\
all CDS of CA & 669 & 66 & 735 & 134,773 & $0.55 \%$ \\
all CDS of WA & 493 & 10 & 503 & 50,680 & $0.99 \%$
\end{tabular}

${ }^{\mathrm{a}} \mathrm{CC}=$ coiled coil; ${ }^{\mathrm{b}} \mathrm{TIR}=$ Toll/Interleukin 1 receptor 


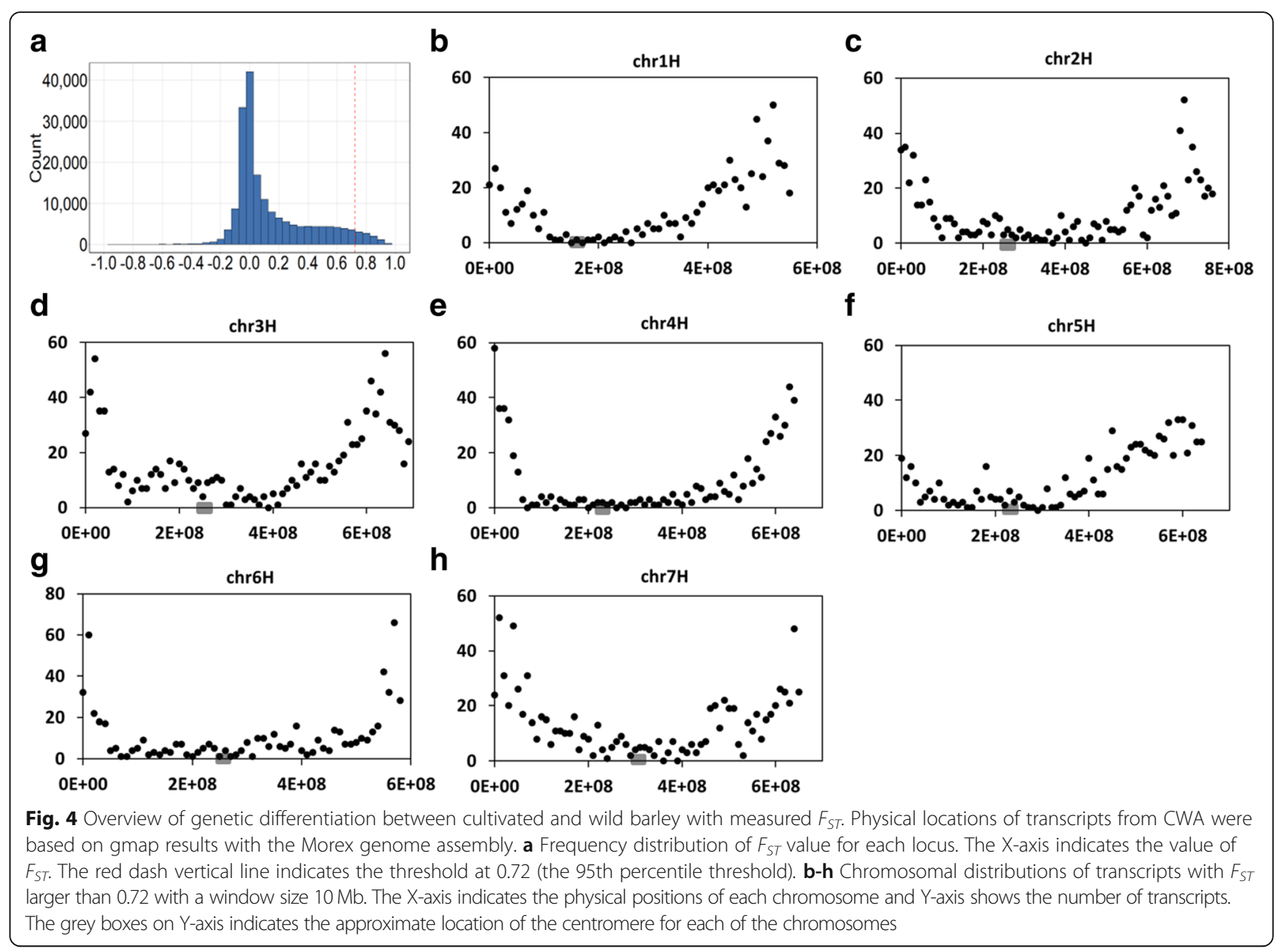

$\geq 90 \%$ identity and $100 \%$ query coverage. The average sequence length of these transcripts is $3687 \mathrm{bp}$. The best matched transcripts from WA are TR133441_c5_g1_(i1, i6) with a maximum of $66 \%$ query coverage. The CDD analysis found that TR100774_c1_g1_i6 of CA (3752 bp, the longest Mla gene transcript of $\mathrm{CA}$ ) has $\mathrm{CC}$ and NB-ARC domains and 5 LRR repeats while the transcript TR133441_c5_g1_i1 (2160 bp, the longest Mla gene transcripts of WA) has only CC and NB-ARC domains without any predicted LRR repeats (Additional file 7: Figure S4).

\section{Discussion}

In the study reported here we constructed a pan-transcriptome of barley by de novo assembling 288 sets of RNA-seq data from 63 genotypes. Approximately 38.2\% of the transcripts from the newly assembled pan-transcriptome were not found in the genome of Morex. The novel transcripts were enriched with genes associated with response to abiotic and biotic stresses. Comparing the cultivated and wild barley genotypes at the pan-transcriptome level found that disease resistance genes are more abundant in the wild barley and they have suffered stronger selective pressures during barley domestication in comparison with genes in other categories.

As a proportion of genes in Morex must also belong to the dispensable genome component, it seems not unreasonable to speculate that the ratio of dispensable genome in barley could be even higher than 38.2\%. However, it is unlikely that all genes from all of genotypes were captured in the RNA-seq data used in this study. Incomplete capture of all genes in individual genotypes invariably lead to an exaggerated proportion of the dispensable genome. Nevertheless, considering the large numbers of genotypes and sequences used in this study, the pan-transcriptome has likely captured the majority of the expressed genes in this species. The proportion of the dispensable genome found here for barley is similar to that for bread wheat $[6,7]$. With the addition of such a large number of novel transcripts on top of those from Morex, the newly assembled pan-transcriptome should facilitate various investigation in barley and its close relatives. We demonstrated as part of this study that, compared with the use of Morex as the reference, four times more SNVs were detected when CWA was used (Fig. 1b). 


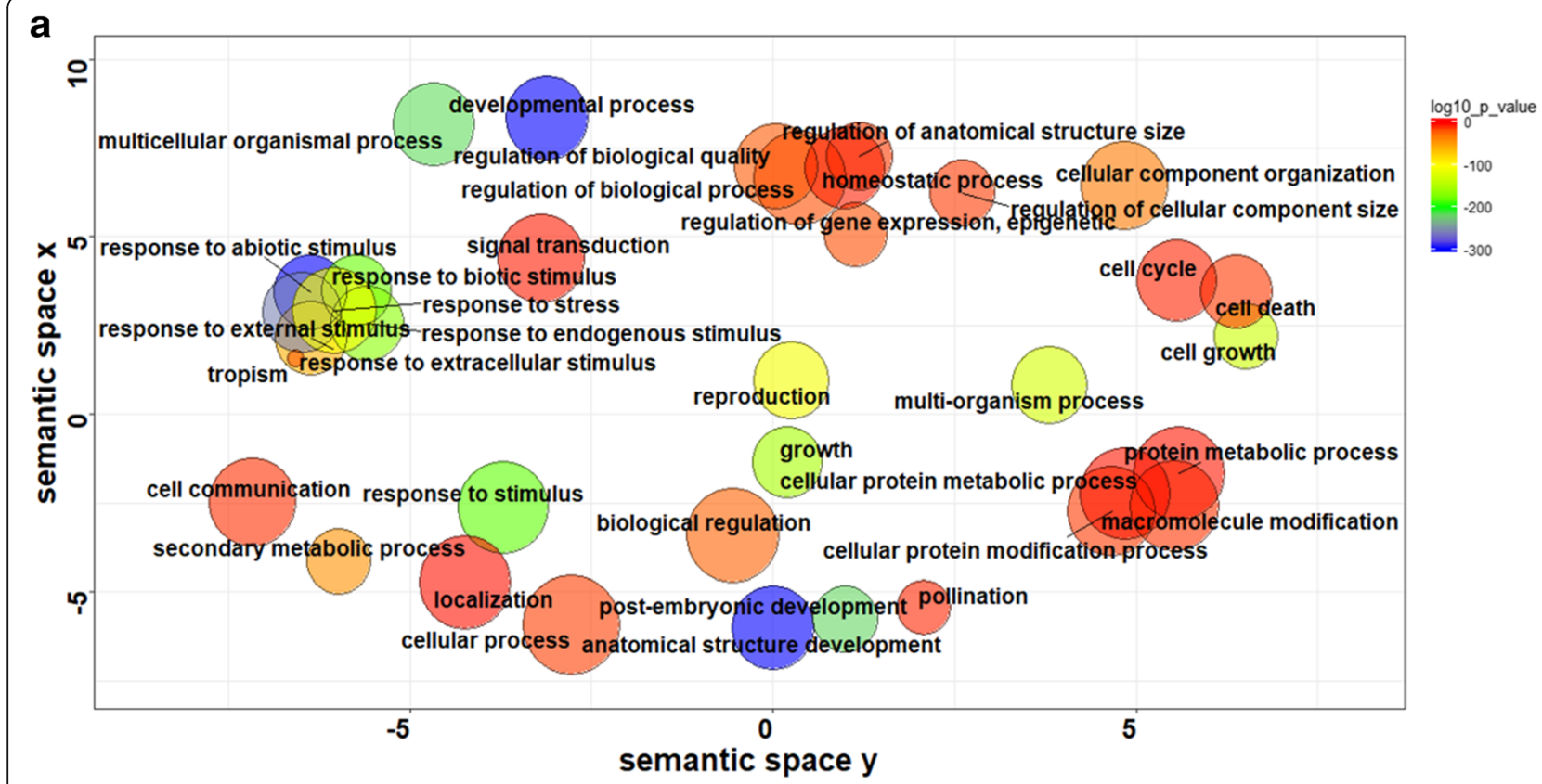

b

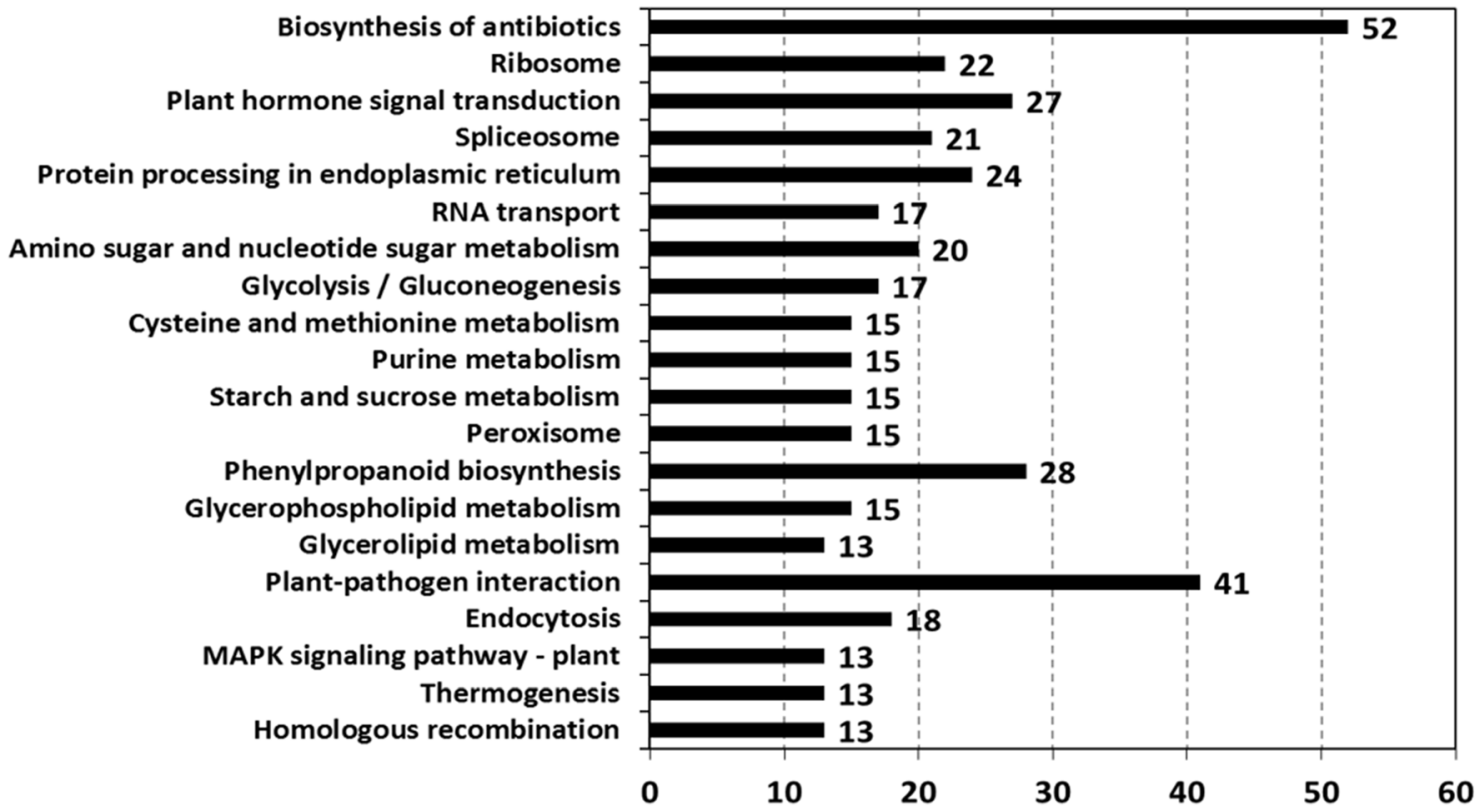

Fig. 5 Functional annotation of genes under strong selective pressures ( $\left.F_{S T}>0.72\right)$. a $G O$ enrichment analysis; (b) count of genes ( $X$-axis) in top 20 enriched KEGG pathways excluding the two global/overview maps (metabolic pathways and biosynthesis of secondary metabolites pathway)

Compared with the cultivated genotypes, wild barley contains substantially more disease resistance genes. This is the case for both the whole transcripts and novel ones only (Fig. 3). Although a larger number of novel CDS are identified from CA than those from WA, the difference unlikely contributed significantly to the comparison as it was conducted using the proportion values. The disease resistance genes include those with NB-ARC, LRR and WAK domains [51-54]. It has been reported that the LRR receptors and WAK are not only regulating plant immunity but also tightly linked to other yield-related genes [55]. NLR-parser analysis also revealed that the wild barley genotypes contained higher percentage of genes conferring disease resistance in comparison with the cultivated ones (Table 1). Importantly, the difference does not seem to be caused by how the samples used in generating the RNA-seq data were obtained. Although the ratio of the disease resistance 
genes was higher in the wild barley genotypes than the cultivated ones, none of the RNA-seq datasets from the former were generated from plants challenged with any biotic pathogen while 16 datasets from cultivated barley genotypes were generated from tissues infected by spider mite (Additional file 1: Table S1). Therefore, the observed difference must, to a large degree, reflect genuine differences between cultivated and wild barley. The genetic differentiation analysis also found that the enriched genes under strong selective pressures are mainly those involved in 'response to stress', 'plant-pathogen interaction', 'phenylpropanoids biosynthesis' and 'plant hormone signal transduction' (Fig. 5). It has been reported the phenylpropanoids can contribute to plant responses towards biotic stimuli and plant hormones can act as signals to trigger defense responses [56, 57]. Thus, these two enriched pathways may help explain why wild barley expresses a higher proportion of disease resistance genes even without pathogen infection. Moreover, the results from the analysis of the Mla gene transcripts provide also further evidence showing that genes related to disease resistance have suffered stronger selection than other gene categories during domestication.

As the increased yield potential is one of the most important changes following domestication and breeding [58], the substantially reduced numbers of genes responsive to diseases in the cultivated genotypes suggest the likelihood that disease resistance can incur costs in yield potential. This likelihood is not different from previous studies showing that resistance often incur physiological costs that reduce host fitness in the absence of the disease in concern in different plant species $[59,60]$. It is believed that such costs can arise because the defence strategy could have harmful pleiotropic effects [61] or because investment in defence requires allocation of limiting resources and hence trade-offs with other traits [59]. Many crop plants have also been characterised by low levels of resistance to pathogen infection [62]. The likelihood that resistance may incur costs that reduce host fitness or yield potential suggests that incorporating a large number of resistance genes into a single genotype may not be an effective breeding strategy. Rather, targeting only genes resistant to major disease for a given environment can be more efficient in breeding cultivars with high yield potential.

\section{Conclusions}

In our study, we constructed a barley pan-transcriptome by using 63 different genotypes. At the pan-transcriptome level, we demonstrated that the disease resistance genes went through stronger selective pressures than other gene categories during barley domestication. With the trade-offs between gaining yield potential and increasing disease resistance during domestication and breeding, we infer that targeting only genes for major diseases for a given environment can be more efficient in variety breeding than incorporating all resistance genes into a specific genotype.

\section{Additional files}

Additional file 1: Table S1. Summary of RNA-seq datasets used in this study. (XLSX 15 kb)

Additional file 2: Table S2. Different filtering criteria for raw sequences of RNA-seq data with different spot lengths (AvgSpotLen). (XLSX 9 kb)

Additional file 3: Figure S1. Functional annotation of novel CDS from CWA. (a) Difference in the percentage of Pfam domains between Morex and CWA (substantially enriched Pfam domain highlighted with *) and (b) Significantly enriched GO terms for biological processes in CWA in comparison with those in Morex $(P<0.05)$. (PNG $83 \mathrm{~kb}$ )

Additional file 4: Table S3. SNVs detected by mapping RNA-seq of 19 barley accessions against either Morex HC + LC or CWA. (XLSX 11 kb)

Additional file 5: Figure S2. Distribution of SNPs along each of the seven chromosomes (a-f). Physical locations of transcripts from CWA were based on the gmap results with the Morex genome assembly. Red line stands for cultivated barley and the blue for wild barley. X-axis shows the physical position of each chromosome and Y-axis indicates the count of SNPs. (PNG $136 \mathrm{~kb}$ )

Additional file 6: Figure S3. PCA results of the 63 genotypes based on the SNP data. (PNG $61 \mathrm{~kb}$ )

Additional file 7: Figure S4. Comparison of functional domains of the longest Mla gene transcript from CA with that from WA. (a) TR100774_C1_ g1_i6 of CA; and (b) TR133441_c5_g1_i1 of WA. (PNG 289 kb)

\section{Abbreviations}

CA: Transcript assembly of cultivated genotypes; CC: Coiled Coil; CDD: Conserved Domains Databases; CDS: Coding DNA sequence; CNL: CC-NBS-LRR; CWA: Transcript assembly of cultivated and wild genotypes; EBI: European Bioinformatics Institute; EMBL: European Molecular Biology Lab; ENA: European Nucleotide Archive; EST: Expressed sequence tag; $F_{S T}$ : Fixation index; GEO: Gene Expression Omnibus; GO: Gene ontology; HC: High-confidence; HSF: Heat shock factor; HSP: Heat shock protein; KEGG: Kyoto Encyclopedia of Genes and Genome; LC: Low-confidence; LEA: Late embryogenesis abundant; LRR: Leucine-rich repeat; Mla: Mildew resistance locus a; NB-ARC: NucleotideBinding adaptor shared by APAF-1, R proteins, and CED-4; NBS: Nucleotidebinding site; NCBI: National Center for Biotechnology Information; ncRNA: Non-coding RNA; NLR: Nucleotide-binding leucine-rich repeat; ORF: Open reading frame; PCA: Principle component analysis; PPR: Pentatricopeptide repeat; SANT: SWI3, ADA2, N-COR, and TFIIIB; SNP: Single nucleotide polymorphism; SNV: Single nucleotide variants; SRA: Short Sequence Read Archive; TIR: Toll/Interleukin1 receptor; TNL: TIR-NBS-LRR; WA: Transcript assembly of wild genotypes; WAK: Wall-associated kinase

\section{Acknowledgements}

We are grateful to Dr. Jonathan Powell and Dr. Louise Thatcher from CSIRO Agriculture \& Food for their critical reading of the manuscript.

\section{Funding}

This work was supported by the Commonwealth Scientific and Industrial Organization (CSIRO), Australia (Project code: R-10191-1). The funding body played no role in designing the study, in collecting, analysing and interpreting the data used, or in writing the manuscript.

Availability of data and materials

Sequence of the de novo assembled transcripts and the mapping results of CWA to Morex genome assembly have been deposited in figshare repository 
(https://figshare.com/s/01af68ada0b889a07759). Other data analysed during this study are included in this manuscript and the additional files.

\section{Authors' contributions}

$\mathrm{YM}$. and $\mathrm{CL}$. conceived and designed the experiments. YM, ML. and JS. performed the data analysis. YM analysed the results and prepared the Figs. YM and $\mathrm{CL}$. wrote the manuscript text. All authors read and approved the final manuscript.

\section{Ethics approval and consent to participate}

Not applicable.

\section{Consent for publication}

Not applicable.

\section{Competing interests}

The authors declare that they have no competing interests.

\section{Publisher's Note}

Springer Nature remains neutral with regard to jurisdictional claims in published maps and institutional affiliations.

\section{Author details}

'CSIRO Agriculture \& Food, 306 Carmody Road, St Lucia, QLD 4067, Australia. ${ }^{2}$ Crop Research Institute of Sichuan Academy of Agricultural Sciences, Chengdu 610066, China.

\section{Received: 13 August 2018 Accepted: 9 December 2018} Published online: 07 January 2019

\section{References}

1. Tettelin H, Masignani V, Cieslewicz MJ, Donati C, Medini D, Ward NL, et al. Genome analysis of multiple pathogenic isolates of Streptococcus agalactiae: implications for the microbial "pan-genome". Proc Natl Acad Sci U S A. 2005;102:13950-5

2. Marroni F, Pinosio S, Morgante M. Structural variation and genome complexity: is dispensable really dispensable? Curr Opin Plant Biol. 2014; 18:31-6.

3. Wing RA. Harvesting rice's dispensable genome. Genome Biol. 2015;16:217.

4. Li YH, Zhou G, Ma J, Jiang W, Jin LG, Zhang Z, et al. De novo assembly of soybean wild relatives for pan-genome analysis of diversity and agronomic traits. Nat Biotechnol. 2014;32:1045-52.

5. Golicz AA, Bayer PE, Barker GC, Edger PP, Kim H, Martinez PA, et al. The pangenome of an agronomically important crop plant Brassica oleracea. Nat Commun. 2016;7:13390.

6. Liu M, Stiller J, Holusova K, Vrana J, Liu DC, Dolezel J, et al. Chromosomespecific sequencing reveals an extensive dispensable genome component in wheat. Sci Rep. 2016;6:36398.

7. Montenegro JD, Golicz AA, Bayer PE, Hurgobin B, Lee H, Chan CKK, et al. The pangenome of hexaploid bread wheat. Plant J. 2017;90:1007-13.

8. Hirsch CN, Foerster JM, Johnson JM, Sekhon RS, Muttoni G, Vaillancourt B, et al. Insights into the maize pan-genome and pan-transcriptome. Plant Cell. 2014;26:121-35

9. Jin M, Liu H, He C, Fu J, Xiao Y, Wang Y, et al. Maize pan-transcriptome provides novel insights into genome complexity and quantitative trait variation. Sci Rep. 2016:6:18936.

10. Sun C, Hu ZQ, Zheng TQ, Lu KC, Zhao Y, Wang WS, et al. RPAN: rice pangenome browser for similar to 3000 rice genomes. Nucleic Acids Res. 2017; 45:597-605.

11. Mayer KX, Waugh R, Langridge P, Close TJ, Wise RP, Graner A, et al. A physical, genetic and functional sequence assembly of the barley genome. Nature. 2012:491:711-6.

12. Mascher M, Gundlach $\mathrm{H}$, Himmelbach A, Beier S, Twardziok SO, Wicker T, et al. A chromosome conformation capture ordered sequence of the barley genome. Nature. 2017;544:427-33.

13. Wang Z, Gerstein M, Snyder M. RNA-Seq: a revolutionary tool for transcriptomics. Nat Rev Genet. 2009;10:57-63.

14. Ziemann M, Kamboj A, Hove RM, Loveridge S, El-Osta A, Bhave M. Analysis of the barley leaf transcriptome under salinity stress using mRNA-Seq. Acta Physiol Plant. 2013;35:1915-24.
15. Huebner S, Korol AB, Schmid KJ. RNA-Seq analysis identifies genes associated with differential reproductive success under drought-stress in accessions of wild barley Hordeum spontaneum. BMC Plant Biol. 2015; $15: 134$

16. Tombuloglu G, Tombuloglu H, Sakcali MS, Unver T. High-throughput transcriptome analysis of barley (Hordeum vulgare) exposed to excessive boron. Gene. 2015;557:71-81.

17. Hill CB, Cassin A, Keeble-Gagnere G, Doblin MS, Bacic A, Roessner U. De novo transcriptome assembly and analysis of differentially expressed genes of two barley genotypes reveal root-zone-specific responses to salt exposure. Sci Rep. 2016;6:31558.

18. Pacak A, Barciszewska-Pacak M, Swida-Barteczka A, Kruszka K, Sega P, Milanowska $K$, et al. Heat stress affects pi-related genes expression and inorganic phosphate deposition/accumulation in barley. Front Plant Sci. 2016:7:926

19. Baker K, Bayer M, Cook N, Dressig S, Dhillon T, Russell J, et al. The lowrecombining pericentromeric region of barley restricts gene diversity and evolution but not gene expression. Plant J. 2014;79:981-92.

20. Dai F, Chen ZH, Wang X, Li Z, Jin G, Wu D, et al. Transcriptome profiling reveals mosaic genomic origins of modern cultivated barley. Proc Natl Acad Sci U S A. 2014;111:13403-8.

21. Chen X, Long H, Gao P, Deng G, Pan Z, Liang J, et al. Transcriptome assembly and analysis of Tibetan hulless barley (Hordeum vulgare L. var. nudum) developing grains, with emphasis on quality properties. PLoS One. 2014;9:e0098144.

22. Zhang Q, Zhang X, Wang S, Tan C, Zhou G, Li C. Involvement of alternative splicing in barley seed germination. PLoS One. 2016;11:e0152824.

23. Comadran J, Kilian B, Russell J, Ramsay L, Stein N, Ganal M, et al. Natural variation in a homolog of Antirrhinum CENTRORADIALIS contributed to spring growth habit and environmental adaptation in cultivated barley. Nat Genet. 2012;44:1388-92.

24. 24. Groenvold L, Schubert M, Sandve SR, Fjellheim S, Hvidsten TR. Comparative transcriptomics reveals lineage specific evolution of cold response in Pooideae. bioRxiv. 2017. https://doi.org/10.1101/151431.

25. Digel B, Pankin A, von Korff M. Global transcriptome profiling of developing leaf and shoot apices reveals distinct genetic and environmental control of floral transition and inflorescence development in barley. Plant Cell. 2015;27: 2318-34.

26. Bahieldin A, Atef A, Sabir JM, Gadalla NO, Edris S, Alzohairy AM, et al. RNASeq analysis of the wild barley $(H$. spontaneum) leaf transcriptome under salt stress. C R Biol. 2015:338:285-97.

27. Cox MP, Peterson DA, Biggs PJ. SolexaQA: at-a-glance quality assessment of Illumina second-generation sequencing data. BMC Bioinformatics. 2010;11:485.

28. Grabherr MG, Haas BJ, Yassour M, Levin JZ, Thompson DA, Amit I, et al. Full-length transcriptome assembly from RNA-Seq data without a reference genome. Nat Biotechnol. 2011;29:644-U130.

29. Schmieder R, Edwards R. Fast identification and removal of sequence contamination from genomic and metagenomic datasets. PLoS One. 2022;6:e17288.

30. Wu TD, Watanabe CK. GMAP: a genomic mapping and alignment program for mRNA and EST sequences. Bioinformatics. 2005:21:1859-75.

31. Li W, Godzik A. Cd-hit: a fast program for clustering and comparing large sets of protein or nucleotide sequences. Bioinformatics. 2006:22:1658-9.

32. McCarthy FM, Gresham CR, Buza TJ, Chouvarine P, Pillai LR, Kumar R, et al. AgBase: supporting functional modeling in agricultural organisms. Nucleic Acids Res. 2011;39:D497-506

33. Tian T, Liu Y, Yan HY, You Q, Yi X, Du Z, et al. agriGO v2.0: a GO analysis toolkit for the agricultural community, 2017 update. Nucleic Acids Res. 2017; 45:W122-9.

34. Supek F, Bosnjak M, Skunca N, Smuc T. REVIGO summarizes and visualizes long lists of gene ontology terms. PLoS One. 2011;6:e0021800.

35. Finn RD, Clements J, Arndt W, Miller BL, Wheeler TJ, Schreiber F, et al. HMMER web server: 2015 update. Nucleic Acids Res. 2015;43:W30-8.

36. Finn RD, Coggill $P$, Eberhardt RY, Eddy SR, Mistry J, Mitchell AL, et al. The Pfam protein families database: towards a more sustainable future. Nucleic Acids Res. 2016:44:D279-85.

37. Steuernagel B, Jupe F, Witek K, Jones JG, Wulff BH. NLR-parser: rapid annotation of plant NLR complements. Bioinformatics. 2015;31:1665-7.

38. Matsumoto T, Tanaka T, Sakai H, Amano N, Kanamori H, Kurita K, et al. Comprehensive sequence analysis of 24,783 barley full-length cDNAs derived from 12 clone libraries. Plant Physiol. 2011;156:20-8. 
39. Zhao Y, Li H, Fang SS, Kang Y, Wu W, Hao YJ, et al. NONCODE 2016: an informative and valuable data source of long non-coding RNAs. Nucleic Acids Res. 2016;44:D203-8.

40. Nawrocki EP, Burge SW, Bateman A, Daub J, Eberhardt RY, Eddy SR, et al, Rfam 12.0: updates to the RNA families database. Nucleic Acids Res. 2015;43: D130-7.

41. Nawrocki EP, Eddy SR. Infernal 1.1: 100-fold faster RNA homology searches. Bioinformatics. 2013;29:2933-5.

42. Langmead B, Salzberg SL. Fast gapped-read alignment with bowtie 2. Nat Methods. 2012;9:357-U354.

43. Li H. A statistical framework for SNP calling, mutation discovery, association mapping and population genetical parameter estimation from sequencing data. Bioinformatics. 2011;27:2987-93.

44. Cingolani P, Platts A, Wang LL, Coon M, Nguyen T, Wang L, et al. A program for annotating and predicting the effects of single nucleotide polymorphisms, SnpEff: SNPs in the genome of Drosophila melanogaster strain $w^{1118}$; iso-2; iso3. Fly. 2012;6:80-92.

45. Danecek P, Auton A, Abecasis G, Albers CA, Banks E, DePristo MA, et al. The variant call format and VCFtools. Bioinformatics. 2011;27:2156-8.

46. Purcell S, Neale B, Todd-Brown K, Thomas L, Ferreira MAR, Bender D, Maller J, Sklar P, de Bakker PIW, Daly MJ, et al. PLINK: a tool set for whole-genome association and population-based linkage analyses. Am J Hum Genet. 2007; 81(3):559-75.

47. Yang J, Lee SH, Goddard ME, Visscher PM. GCTA: a tool for genome-wide complex trait analysis. Am J Hum Genet. 2011;88(1):76-82.

48. Jorgensen JH. Genetics of powdery mildew resistance in barley. Crit Rev Plant Sci. 1994;13(1):97-119.

49. Seeholzer S, Tsuchimatsu T, Jordan T, Bieri S, Pajonk S, Yang W, Jahoor A, Shimizu KK, Keller B, Schulze-Lefert P. Diversity at the Mla powdery mildew resistance locus from cultivated barley reveals sites of positive selection. Mol Plant-Microbe Interact. 2010;23(4):497-509.

50. Marchler-Bauer A, Bo Y, Han L, He J, Lanczycki CJ, Lu S, Chitsaz F, Derbyshire MK, Geer RC, Gonzales NR, et al. CDD/SPARCLE: functional classification of proteins via subfamily domain architectures. Nucleic Acids Res. 2017;45(D1): D200-3.

51. Dievart A, Clark SE. LRR-containing receptors regulating plant development and defense. Development. 2004;131:251-61.

52. Van Ooijen G, Mayr G, Kasiem MA, Albrecht M, Cornelissen BC, Takken FW. Structure-function analysis of the NB-ARC domain of plant disease resistance proteins. J Exp Bot. 2008;59:1383-97.

53. Zuo WL, Chao Q, Zhang N, Ye JR, Tan GQ, Li BL, et al. A maize wallassociated kinase confers quantitative resistance to head smut. Nat Genet. 2015;47:151-7.

54. Delteil A, Gobbato E, Cayrol B, Estevan J, Michel-Romiti C, Dievart A, et al. Several wall-associated kinases participate positively and negatively in basal defense against rice blast fungus. BMC Plant Biol. 2016;16:17.

55. Ning Y, Liu W, Wang GL. Balancing immunity and yield in crop plants. Trends Plant Sci. 2017:22:1069-79.

56. Vogt T. Phenylpropanoid biosynthesis. Mol Plant. 2010;3(1):2-20.

57. Yang DL, Yong $\mathrm{Y}, \mathrm{He} Z \mathrm{ZH}$. Roles of plant hormones and their interplay in rice immunity. Mol Plant. 2013;6(3):675-85.

58. Preece C, Livarda A, Christin PA, Wallace M, Martin G, Charles M, et al. How did the domestication of Fertile Crescent grain crops increase their yields? Funct Ecol. 2017;31:387-97.

59. Tian $\mathrm{D}$, Traw MB, Chen JQ, Kreitman M, Bergelson J. Fitness costs of R-genemediated resistance in Arabidopsis thaliana. Nature. 2003;423:74-7.

60. Horns F, Hood ME. The evolution of disease resistance and tolerance in spatially structured populations. Ecology and Evolution. 2012;2:1705-11.

61. Parker MA. The pleiotropy theory for polymorphism of disease resistance genes in plants. Evolution. 1990;44:1872-5.

62. Cordova-Campos O, Adame-Alvarez RM, Acosta-Gallegos JA, Heil M. Domestication affected the basal and induced disease resistance in common bean (Phaseolus vulgaris). Eur J Plant Pathol. 2012;134:367-79.

\section{Ready to submit your research? Choose BMC and benefit from:}

- fast, convenient online submission

- thorough peer review by experienced researchers in your field

- rapid publication on acceptance

- support for research data, including large and complex data types

- gold Open Access which fosters wider collaboration and increased citations

- maximum visibility for your research: over $100 \mathrm{M}$ website views per year

At BMC, research is always in progress.

Learn more biomedcentral.com/submissions 